\title{
On Normals and Control Nets
}

\author{
I. Ginkel ${ }^{1}$, J. Peters ${ }^{2}$, and G. Umlauf ${ }^{1}$ \\ 1 University of Kaiserslautern, Germany ginkel |umlauf@informatik.uni-kl.de \\ ${ }^{2}$ University of Florida, Gainesville, FL, USA jorg@cise.ufl.edu
}

\begin{abstract}
This paper characterizes when the normals of a spline curve or spline surface lie in the more easily computed cone of the normals of the segments of the spline control net.
\end{abstract}

\section{Introduction}

Since, for splines, subdivision amounts to averaging, would not the limit normal of a spline curve or surface be a linear, even convex combination of the normals of the segments of the spline control net? In this introduction and in Section 2 we present some evidence that supports this conjecture for curves and functions. Section 3 discusses the case of box-spline surfaces.

Such an investigation is practically useful, since an affirmative answer to the question would allow substituting a simpler computation of bounds on the control structure for complex, exact bounds on the corresponding nonlinear curve or surface. Such bounds play a significant role in curve and surface intersection algorithms and in efficient or high-quality rendering.

For a planar, polynomial curve segment in B-spline or Bézier form, the cone spanned by the perpendiculars to the control segments encloses the cone of the curve normals. This follows from

(i) the coefficients of the hodograph (the derivative of the parametrization) are the scaled differences of the coefficients of the curve parametrization,

(ii) the hodograph is a convex combination of its coefficients and

(iii) the normal to the curve segment is perpendicular to its hodograph.

In symbols, we consider a planar curve

$$
\mathbf{c}(t):=\sum_{i} \mathbf{c}_{i} b_{i}(t), \quad \mathbf{c}_{i} \in \mathbb{R}^{2}
$$

in terms of Bernstein polynomials or B-splines $b_{i}(t)$ of degree $d$. Then $\perp(x, y)=$ $(-y, x)$ is the normal direction $\mathbf{n}(\mathbf{c}, t)$ of $\mathbf{c}$ at $t$, and we may define

$$
\Delta_{i}(\mathbf{c}):=\left\{\begin{array}{l}
d\left(\mathbf{c}_{i+1}-\mathbf{c}_{i}\right), \text { if } i \in \mathrm{I}:=\{0, \ldots, n-1\} \\
0, \text { else. }
\end{array}\right.
$$


The proof of the assertion is

$$
\begin{aligned}
\mathbf{n}(\mathbf{c}, t) & =\perp\left(\mathbf{c}^{\prime}(t)\right)=\perp\left(\sum_{i} \Delta_{i}(\mathbf{c}) b_{i}(t)\right)=\sum_{i} \perp\left(\Delta_{i}(\mathbf{c})\right) b_{i}(t) \\
& \in \operatorname{cone}\left(\perp\left(\Delta_{i}(\mathbf{c})\right)\right)_{i \in \mathrm{I}} .
\end{aligned}
$$

Note that the basic idea generalizes to curves in higher-dimensional spaces and characterizes the normal hyperplanes perpendicular to the tangent cone spanned by the differences of the control polygon.

\section{Curves and Bivariate Functions}

We generalize the earlier argument to rational planar curves of the form

$$
\mathbf{r}(t):=\frac{\sum_{i} w_{i} \mathbf{r}_{i} b_{i}(t)}{\sum_{i} w_{i} b_{i}(t)}, \quad \mathbf{r}_{i} \in \mathbb{R}^{2},
$$

with $w_{i}>0$ : the cone spanned by the perpendiculars to the control segments encloses the cone of the curve normals.
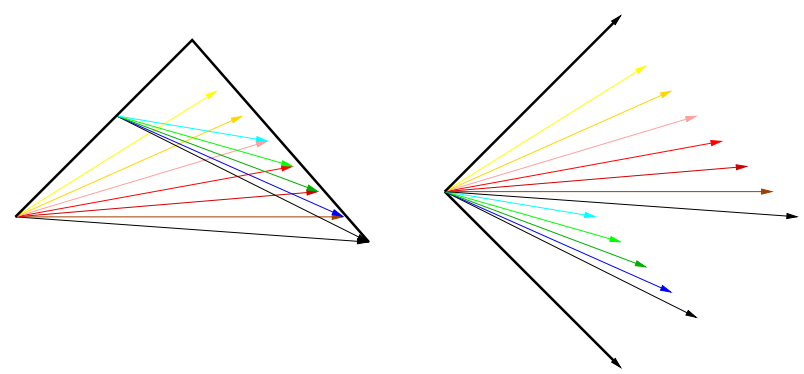

Fig. 1. The differences of intermediate points generated by de Boor's algorithm lie in the cone spanned be the differences $\Delta_{i}(\mathbf{r}), i \in \mathrm{I}$.

Lemma 1. Let $\mathbf{n}(\mathbf{r}, t)$ be the normal to the rational Bézier curve segment $\mathbf{r}$ and $\rho:=\sum_{i} \mathbf{r}_{i} b_{i}$. Then

$$
\mathbf{n}(\mathbf{r}, t) \in \operatorname{cone}\left(\perp\left(\Delta_{i}(\rho)\right)\right)_{i \in \mathbf{l}}
$$

Proof. Both the rational de Casteljau and the de Boor algorithm use only pairwise convex combinations to compute intermediate points $\rho_{i}^{j}, j=0, \ldots, d, i=$ $0, \ldots, j$, when written in non-homogeneous space (see e.g. [1]), For non-negative weights, the differences of intermediate points lie therefore in the cone spanned by the differences $\Delta_{i}(\rho), i \in \mathrm{I}$ (Figure 1) and the tangent of $\mathbf{r}$ at parameter $t$ is 
the scaled difference $\rho_{1}^{d-1}-\rho_{0}^{d-1}[2,3]$ of the affine intermediate control points. Finally, the normal direction $\mathbf{n}(\mathbf{r}, t)$ of $\mathbf{r}$ at $t$ is perpendicular to the tangent at $t$.

Similarly, for a planar $C^{1}$ subdivision curve generated by a binary subdivision scheme with weights $\alpha_{i}$

$$
\begin{aligned}
\mathbf{s}_{2 i}^{\prime} & =\sum_{j} \alpha_{2 i-2 j} \mathbf{s}_{j}, \\
\mathbf{s}_{2 i+1}^{\prime} & =\sum_{j} \alpha_{2 i+1-2 j} \mathbf{s}_{j},
\end{aligned}
$$

whose difference scheme uses only convex combinations (see [4]), the cone spanned by the perpendiculars to the control segments encloses the cone of the curve normals

$$
\mathbf{n}(\mathbf{s}, t) \in \operatorname{cone}\left(\perp\left(\Delta_{i}(\mathbf{s})\right)\right)_{i \in \mathbf{I}}
$$

This follows from

(i) the divided differences of control points of a refined control polygon lie in the cone of divided differences of control points of the coarse control polygon,

(ii) the divided differences converge towards the scaled tangents,

(iii) the normal direction $\mathbf{n}(\mathbf{s}, t)$ of $\mathbf{s}$ at $t$ is perpendicular to the tangent at $t$.
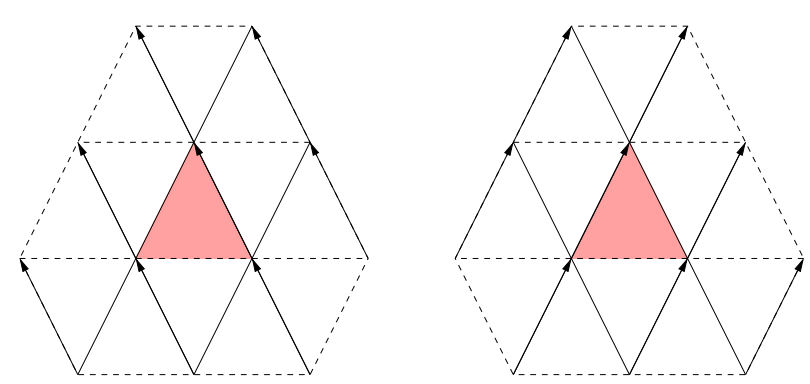

Fig. 2. Control points $p_{\mathrm{i}}, \mathrm{i} \in \mathrm{I}$ (the set of all 12 line intersections) defining a degree 4 three-direction box-spline parametrized over the central, shaded triangle. The arrows indicate two (affinely skewed, box-spline averaging) directions $\mathrm{e}_{1}$ and $\mathrm{e}_{2}$ contributing to the partial derivatives of the surface piece. Note that, in each case, four of the twelve differences are zero since they do not contribute to the derivative.

Let us now consider a (uniform box-)spline (see [5]) in two variables $\left(\mathrm{u}_{1}, \mathrm{u}_{2}\right)=\mathrm{u}$,

$$
p(\mathrm{u}):=\sum_{\mathrm{i}} p_{\mathrm{i}} b_{\mathrm{i}}(\mathrm{u})
$$


with coefficients $p_{\mathrm{i}} \in \mathbb{R}$ and $\mathrm{i}=\left(\mathrm{i}_{1}, \mathrm{i}_{2}\right) \in \mathbb{Z}^{2}$, a multi-index. One polynomial piece of such a spline is a linear combination of nodal or box-spline functions $b_{\mathrm{i}}, \mathrm{i} \in \mathrm{I}$. In particular, we consider the three-direction box spline with directions $\Xi:=\left[\begin{array}{llllll}1 & 1 & 0 & 0 & 1 & 1 \\ 0 & 0 & 1 & 1 & 1 & 1\end{array}\right]$. A polynomial piece $p$ is defined by a submesh consisting of all triangles (point- or edge-) adjacent to a central triangle (see Figure 2). Two derivatives in the generating directions, without loss of generality, $e_{1}:=(1,0)$ and $e_{2}:=(0,1)$, can be obtained by differencing control points in these directions. We define

$$
\Delta_{k, \mathrm{i}}(p):=\left\{\begin{array}{l}
d\left(p_{\mathrm{i}+\mathrm{e}_{k}}-p_{\mathrm{i}}\right), \text { if both } \mathrm{i} \in \mathrm{I} \text { and } \mathrm{i}+\mathrm{e}_{k} \in \mathrm{I} \\
0 \text { else. }
\end{array}\right.
$$

Then the same relation holds as for planar curves.

Lemma 2. The normal of a polynomial piece $p$ of a bivariate box-spline function with averaging matrix $\Xi$ is in the normal cone spanned by the control facet normals with vertices $p_{\mathrm{i}}, \mathrm{i} \in \mathrm{I}$.

Proof. By definition, the normal direction is

$$
\begin{aligned}
\mathbf{n}\left(\left(\begin{array}{c}
\mathrm{u}_{1} \\
\mathrm{u}_{2} \\
p
\end{array}\right), \mathrm{u}\right) & :=\frac{\partial\left(\begin{array}{c}
\mathrm{u}_{1} \\
\mathrm{u}_{2} \\
p
\end{array}\right)}{\partial \mathrm{u}_{1}} \times \frac{\partial\left(\begin{array}{c}
\mathrm{u}_{1} \\
\mathrm{u}_{2} \\
p
\end{array}\right)}{\partial \mathrm{u}_{2}} \\
& =\sum_{\mathrm{i}}\left(\begin{array}{c}
1 \\
0 \\
\Delta_{1, \mathrm{i}}(p)
\end{array}\right) b_{\mathrm{i}}(\mathrm{u}) \times \sum_{\mathrm{i}}\left(\begin{array}{c}
0 \\
1 \\
\Delta_{2, \mathrm{i}}(p)
\end{array}\right) b_{\mathrm{i}}(\mathrm{u}) \\
& =\left(\begin{array}{c}
1 \\
0 \\
\sum_{\mathrm{i}} \Delta_{1, \mathrm{i}}(p) b_{\mathrm{i}}(\mathrm{u})
\end{array}\right) \times\left(\begin{array}{c}
0 \\
1 \\
\sum_{\mathrm{i}} \Delta_{2, \mathrm{i}}(p) b_{\mathrm{i}}(\mathrm{u})
\end{array}\right) \\
& =\sum_{\mathrm{i}}\left(\begin{array}{c}
-\Delta_{1, \mathrm{i}}(p) \\
-\Delta_{2, \mathrm{i}}(p) \\
1
\end{array}\right) b_{\mathrm{i}}(\mathrm{u}) \\
& \in \operatorname{cone}\left(\left(\begin{array}{c}
-\Delta_{1, \mathrm{i}}(p) \\
-\Delta_{2, \mathrm{i}}(p) \\
1
\end{array}\right)\right)_{\mathrm{i} \in \mathrm{I}},=\operatorname{cone}\left(\Delta_{1, \mathrm{i}}\left(\begin{array}{c}
\mathrm{u}_{1} \\
\mathrm{u}_{2} \\
p
\end{array}\right) \times \Delta_{2, \mathrm{i}}\left(\begin{array}{c}
\mathrm{u}_{1} \\
\mathrm{u}_{2} \\
p
\end{array}\right)\right)_{\mathrm{i} \in \mathrm{I} .} .
\end{aligned}
$$

The last expressions are the normals of the control facets.

Here, we picked the three-direction box-spline since the control facets have a unique normal, as opposed to the control net of a tensor-product spline.

\section{Box-spline surfaces}

The examples of curves in one and functions in two variables suggest that the cone of normal directions $\mathbf{n}(\mathbf{p}, \mathbf{u}), \mathbf{u} \in U$, of a spline surface patch $\mathbf{p}$ is in the cone of the normals of its spline control polyhedron of $\mathbf{p}$. To test this hypothesis, we consider again the three-direction box splines with direction matrix $\Xi:=$ $\left[\begin{array}{llllll}1 & 1 & 0 & 0 & 1 & 1 \\ 0 & 0 & 1 & 1 & 1 & 1\end{array}\right]$. 
Lemma 3. The normal of a polynomial piece $\mathbf{p}$ of a bivariate parametric boxspline surface with averaging matrix $\Xi$ is in the normal cone spanned by the cross products of each difference in the direction $\mathrm{e}_{1}$ with each difference in the direction $\mathrm{e}_{2}$ of control points $\mathbf{p}_{\mathbf{i}}, \mathrm{i} \in \mathrm{I}$.

Proof. Since the cross product results in a polynomial of degree $2 d-2$ and the box-spline functions $b_{\mathrm{i}}$ form a non-negative partition of 1 , we can bound the normal by

$$
\begin{aligned}
\mathbf{n}(\mathbf{p}, \mathrm{u}) & :=\frac{\partial \mathbf{p}}{\partial \mathrm{u}_{1}} \times \frac{\partial \mathbf{p}}{\partial \mathrm{u}_{2}}=\sum_{\mathrm{i} \in \mathrm{I}} \Delta_{1, \mathrm{i}}(\mathbf{p}) b_{\mathrm{i}}(\mathrm{u}) \times \sum_{\mathbf{j} \in \mathrm{I}} \Delta_{2, \mathrm{j}}(\mathbf{p}) b_{\mathrm{j}}(\mathrm{u}) \\
& \in \operatorname{cone}\left(\Delta_{1, \mathrm{i}}(\mathbf{p}) \times \Delta_{2, \mathrm{j}}(\mathbf{p})\right)_{\mathrm{i} \in \mathrm{I}, \mathrm{j} \in \mathrm{I}} .
\end{aligned}
$$

Lemma 3 indicates that a much larger set, the set of cross products of all edge vectors of the control net in the direction $\mathrm{e}_{1}$ with all edge vectors in the direction $\mathrm{e}_{2}$ generates a cone that includes the limit normals of the patch. This raises the question, whether such a larger set is also necessary.
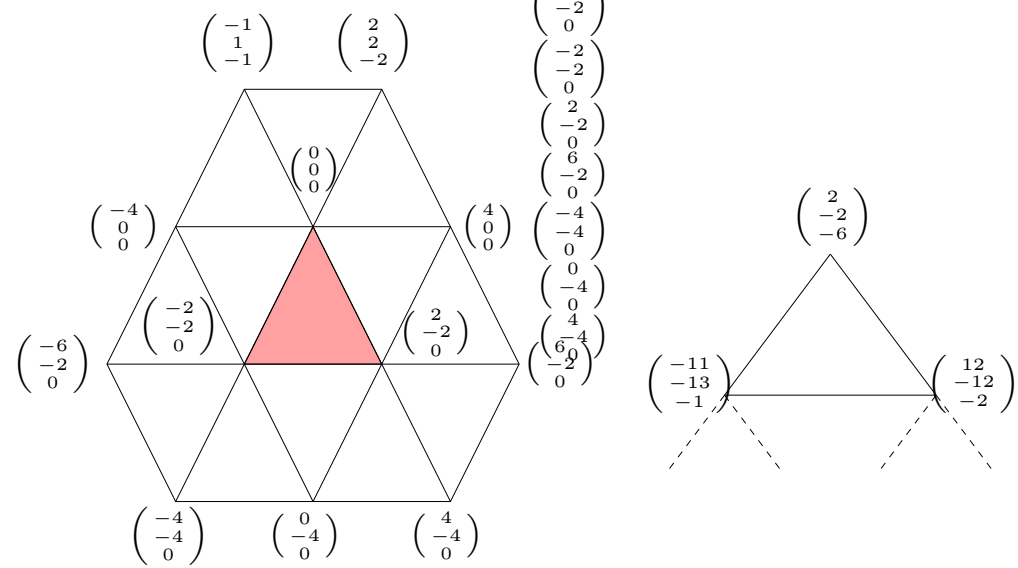

Fig. 3. (left) A three-direction box-spline control net. Only the top two coefficients have a non-zero $z$-component. The $(x, y)$ coordinates of all coefficients, except the top left, are evenly distributed. For simplicity, the top left coefficient is the average of the top right and $(-4,0,0)^{T}$. (right) Bézier control points (scaled by 24) corresponding to the tip of the triangular patch near $(0,0,0)$.

The choice of coefficients in Figure 3, left, shows that it is not enough to just consider the normals of the control facets.

Lemma 4. The normal of a polynomial piece $\mathbf{p}$ of a box-spline surface with averaging matrix $\Xi$ is in general not in the normal cone spanned by the control facet normals with vertices $p_{\mathrm{i}}, \mathrm{i} \in \mathrm{I}$.

Proof. To certify the control net as a counterexample, we use the fact that the three-direction patch $\mathbf{p}$ can alternatively be expressed in terms of Bézier coefficients (see e.g. [6,7] for explicit formulas). Figure 3,right, shows the Bézier 
coefficients near the top point $(0,0,0)$ corresponding to $\mathbf{0}$. From these it is easy to compute the normal. (Alternatively, we could have used the explicit formula for normals of Loop subdivision surfaces.) Explicitly, with "*" indicating unimportant values,

$$
\mathbf{n}(\mathbf{p}, \mathbf{0})=\left(\begin{array}{c}
-13 \\
-11 \\
5
\end{array}\right) \times\left(\begin{array}{c}
10 \\
-10 \\
4
\end{array}\right)=\left(\begin{array}{l}
6 \\
* \\
*
\end{array}\right),
$$

i.e. the normal at the tip of the triangular patch has a nonzero $x$-component, while the facet normals lie in the $x=0$ plane.

Note that the counterexample crucially depends on non-functional data.

What is the flaw in the argument that, since subdivision amounts to averaging, the limit normals are a linear combination of the initial normals? One flaw is that the normal is bilinear with respect to the action of the subdivision matrices. Hence, we should not expect to find a linear averaging scheme for the normal. Indeed the following lemma shows that, in general, no subdivision scheme exists for the normals.

Lemma 5. No linear subdivision scheme exists for the normals of the threedirection box-spline with direction matrix $\Xi$.

Proof. We perform one subdivision step using the box-spline subdivision rules (see e.g. [8]) on the data of Figure 3, left, resulting in the control net shown in Figure 4. Again, we consider the facet normals at the apex of the central triangle. It has normal direction

$$
\left(\left(\begin{array}{c}
16 \\
0 \\
-2
\end{array}\right)-\left(\begin{array}{c}
1 \\
-1 \\
-3
\end{array}\right)\right) \times\left(\left(\begin{array}{c}
8 \\
-8 \\
0
\end{array}\right)-\left(\begin{array}{c}
1 \\
-1 \\
-3
\end{array}\right)\right)=\left(\begin{array}{c}
15 \\
1 \\
1
\end{array}\right) \times\left(\begin{array}{c}
7 \\
-7 \\
3
\end{array}\right)=\left(\begin{array}{c}
10 \\
* \\
*
\end{array}\right) .
$$

Since the normal direction has a nonzero $x$-component, it is not in the space spanned by the facet normals of the original control mesh.

\section{Generalizations}

At least one further investigation deserves attention. Since the cross products, of all edges of the control net of one directional derivative with all edges of another, forms a cone that contains the normals of the regular part of a Loop subdivision surface [8], can a similar statement be made for the neighborhood of extraordinary points of a subdivision surface?

Acknowledgement This research was support in part by NSF under grants DMI-0400214 and CCF-0430891.

\section{References}

1. Boehm, W.: An affine representation of de Casteljau's and de Boor's rational algorithms. Computer Aided Geometric Design 10 (1993) 175-180

2. Floater, M.: Derivatives of rational Bézier curves. Computer Aided Geometric Design 9 (1992) 161-174 


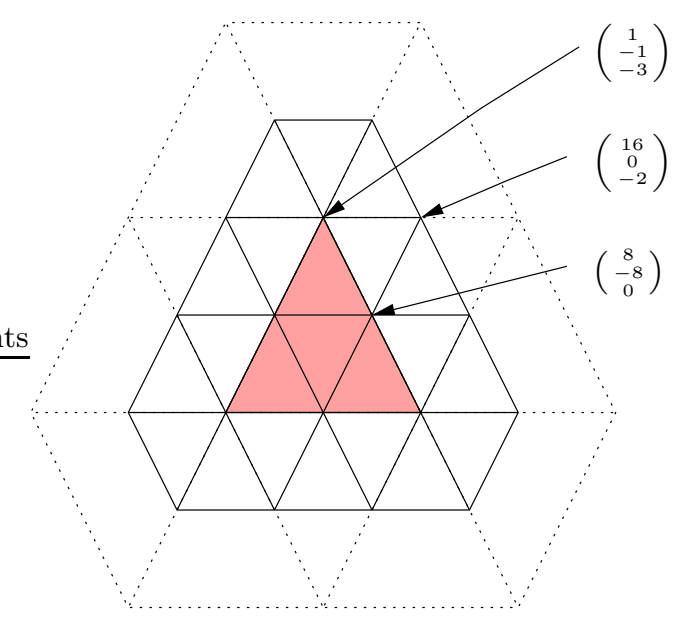

Fig. 4. Coefficients (scaled by 8 ) of the once-refined control net.

3. Floater, M.: Evaluation and properties of the derivative of a NURBS curve. In Lyche, T., Schumaker, L.L., eds.: Mathematical Methods in CAGD, Boston, Academic Press (1992) 261-274

4. Dyn, N.: Subdivision schemes in CAGD. In Light, W., ed.: Advances in Numerical Analysis. Volume II Wavelets, Subdivision Algorithms, and Radial Basis Functions., Oxford Science Publications (1992) 37-104

5. de Boor, C., Höllig, K., Riemenschneider, S.: Box splines. Volume 98 of Applied Mathematical Sciences. Springer, New York (1993)

6. Böhm, W.: Generating the Bézier points of triangular splines. In Barnhill, R., Bhm, W., eds.: Surfaces in Computer Aided Geometric Design, North-Holland Publishing Company, (1983) 77-91

7. Peters, J., Shiue, L.: Combining 4- and 3-direction subdivision. ACM Transactions on Graphics 23 (2004) 980-1003

8. Loop, C.T.: Smooth subdivision surfaces based on triangles. Master's thesis, Department of Mathematics, University of Utah (1987)

9. Saito, T., Wang, G., Sederberg, T.: Hodographs and normals of rational curves and surfaces. Computer Aided Geometric Design 12 (1995) 417-430 